\title{
OPEN Community composition and functional prediction of prokaryotes associated with sympatric sponge species of southwestern Atlantic coast
}

\author{
C. C. P. Hardoim ${ }^{1 凶}$, A. C. M. Ramaglia ${ }^{1}$, G. Lôbo-Hajduํㄹ \& M. R. Custódio ${ }^{3}$
}

Prokaryotes contribute to the health of marine sponges. However, there is lack of data on the assembly rules of sponge-associated prokaryotic communities, especially for those inhabiting biodiversity hotspots, such as ecoregions between tropical and warm temperate southwestern Atlantic waters. The sympatric species Aplysina caissara, Axinella corrugata, and Dragmacidon reticulatum were collected along with environmental samples from the north coast of São Paulo (Brazil). Overall, 64 prokaryotic phyla were detected; 51 were associated with sponge species, and the dominant were Proteobacteria, Bacteria (unclassified), Cyanobacteria, Crenarchaeota, and Chloroflexi. Around $64 \%$ and $89 \%$ of the unclassified operational taxonomical units (OTUs) associated with Brazilian sponge species showed a sequence similarity below $97 \%$, with sequences in the Silva and NCBI Type Strain databases, respectively, indicating the presence of a large number of unidentified taxa. The prokaryotic communities were species-specific, ranging $56 \%-80 \%$ of the OTUs and distinct from the environmental samples. Fifty-four lineages were responsible for the differences detected among the categories. Functional prediction demonstrated that Ap. caissara was enriched for energy metabolism and biosynthesis of secondary metabolites, whereas $D$. reticulatum was enhanced for metabolism of terpenoids and polyketides, as well as xenobiotics' biodegradation and metabolism. This survey revealed a high level of novelty associated with Brazilian sponge species and that distinct members responsible from the differences among Brazilian sponge species could be correlated to the predicted functions.

The phylum Porifera consists of sessile and filter-feeding animal communities, which are among the oldest living Metazoans, dating back to approximately $700 \mathrm{Myr}^{2}{ }^{1}{ }^{1}$. The class Demospongiae encompasses $85 \%$ of the 9345 valid sponge species described thus far $^{2}$. One of the challenges of in studying these animals is that several species lack morphological features that assist classical taxonomy. For instance, the families Aplysinidae and Axinellidae are notoriously difficult to identify due to the lack of diagnostic morphological features ${ }^{3-6}$. Therefore, DNA barcoding has been used to aid species identification ${ }^{7}$. Because of their intrinsic characteristics, sponges are known to perform several services contributing to the functioning and health of benthic ecosystems ${ }^{8,9}$. Marine sponges also harbour diverse and complex prokaryotic communities ${ }^{9,10}$, and in certain cases, up to $38 \%$ of the sponge wet weight is composed of bacterial cells ${ }^{11}$. The most comprehensive study performed so far detected between 41 and 72 recognised and candidate prokaryotic phyla associated with 269 sponge species encountered worldwide, but not from the Brazilian coast ${ }^{10,12}$.

The field of sponge microbiology in Brazil is still underdeveloped. The Brazilian marine coast encompasses approximately $7367 \mathrm{~km}$ and currently has 597 sponge species distributed within the four classes, which correspond to approximately $6 \%$ of the global sponge diversity ${ }^{13}$. Among them, 113 sponge species are endemic to the Brazilian coast ${ }^{13}$. The number of registers in this region is constantly increasing due to new records and

${ }^{1}$ Institute of Biosciences, São Paulo State University, Coastal Campus of São Vicente, São Paulo, Brazil. ${ }^{2}$ Department of Genetic, Biology Institute Roberto Alcântara Gomes, Rio de Janeiro State University, Rio de Janeiro, Brazil. ${ }^{3}$ Department of Physiology, Center for Marine Biology, Biosciences Institute and NP-Biomar, São Paulo University, São Paulo, Brazil. ${ }^{\varpi}$ email: cristianehardoim@gmail.com 
species $^{14-16}$. However, only four prior studies have assessed the prokaryotic communities associated with the Brazilian marine sponges, which were performed with species from the coast of Rio de Janeiro using less robust techniques ${ }^{17-20}$.

To assess the importance of deterministic components for prokaryotic community assembly in southwestern Atlantic, three common sponge species from São Paulo state were investigated. This coastal area has approximately $600 \mathrm{~km}$ of extension, divided into littoral south and north, and encompasses the transition between tropical and warm temperate southwestern Atlantic marine ecoregions ${ }^{21}$. This region is considered one of the most significant benthic biodiversity hotspots and ecosystem services globally but is relatively unknown. It is characterised by microtides, a transition between low to high productivity, where sponges are one of the most important eco-engineers. However, anthropogenic threats, such as urbanisation, invasive species, climate change, and mining, are affecting the three-dimensional living structures offered by sessile animals ${ }^{22}$. The city of São Sebastião is located on the north coast and hosts over 70 sponge species described, belonging to the classes Calcarea and Demosponges ${ }^{23}$, and might be considered a local hotspot of sponge diversity in Brazil. The sympatric sponges Aplysina caissara (Pinheiro \& Hajdu, 2001), Dragmacidon reticulatum (Ridley \& Dendy, 1886), and Axinella corrugata (George \& Wilson, 1919), along with the environmental samples (seawater and sediment) were collected from this region. Aplysina caissara has a narrow distribution and is endemic to southern and southeastern Brazil ${ }^{4}$. Even though a sponge with similar characteristics as Ap. caissara was discovered in the Guyana shelf, certain features made the specimen identification difficult ${ }^{24}$; hence, we considered its original distribution. Ax. corrugata and D. reticulatum are widely distributed along the Brazilian coast and the Caribbean $\mathrm{Sea}^{2}$. The main questions in this study were $(\boldsymbol{i})$ whether DNA barcoding was capable of separating the sponge species, $(\mathbf{i i})$ whether the prokaryotic communities associated with the sponge species can be distinguished from the ones detected in the environmental samples, (iii) whether the sponge species exhibited host specificity, (iv) which prokaryotic lineages are responsible for the variability detected in the categories, and $(\boldsymbol{v})$ which predicted functional patterns are enriched in each category.

\section{Results}

Sponge barcoding. Analysis of 364 bp-long sequences of the mitochondrial cytochrome b (cob) obtained from all 15 specimens showed no intraspecific variations among our sequences of Ap. caissara, Ax. corrugata, and D. reticulatum. A genetic distance ( $p$-distance) of $0.82 \%$ was observed among Ap. caissara and other Aplysina species, whereas $18.13 \%$ was detected between Ax. corrugata from GenBank and our sequences. For Ap. caissara and $D$. reticulatum, these were the first cob sequences reported and, as such, no prior sequence of this gene was available from the National Center for Biotechnology Information (NCBI) for comparison. Phylogenetic reconstructions based on Maximum Likelihood and Bayesian inferences indicated that each sponge species formed a robust cluster (Fig. 1), suggesting that the cob gene is efficient in differentiating Ap. caissara from other Aplysina species.

Analysis of the 16S rRNA gene. A total of 3,814,873 V4-region of the 16S rRNA gene sequences were obtained on an Illumina MiSeq platform. After denoising, quality filtering, and removal of chimera and undesirables, a total of 2,950,757 16S rRNA sequences were further analysed with Mothur v.1.44. Then, singletons were also removed from the dataset resulting in $2,865,820$ sequences that were further rarefied to the same library depth of 70,314 sequences, resulting in 1,757,850 sequences (Supplementary Table S2). These were assigned to 43,947 OTUs at $97 \%$ sequence similarity.

Prokaryotic alpha diversity. The rarefaction curve demonstrated that except for Ap. caissara and sediment, all the other categories reached a plateau with the sequence depth used (Supplementary Fig. S1). Sediment presented the highest observed CHAO richness, followed by seawater, Ap. caissara, Ax. corrugata, and D. reticulatum (Table 1). Analysis of variance (ANOVA) revealed that the categories differed from each other, and this difference was detected between each sponge species and sediment as well as seawater and sediment $(p<0.001)$, (Supplementary Table S3). Sediment also showed the highest estimated CHAO richness, followed by seawater, Ap. caissara, Ax. corrugata, and D. reticulatum. These categories were significantly different according to ANOVA analysis, and it was detected between the categories sediment and each sponge species, seawater and Ax. corrugata, seawater and D. reticulatum, and seawater and sediment $(p<0.001)$, Ap. caissara and D. reticulatum, and seawater and Ap. caissara $(p<0.01)$ (Supplementary Table S3). For the ACE index, the highest richness was observed in sediment, followed by seawater, Ap. caissara, Ax. corrugata, and D. reticulatum (Table 1). These categories differed significantly according to ANOVA. This dissimilarity was registered between sediment and each sponge species, seawater and Ax. corrugata, seawater and D. reticulatum, and seawater and sediment $(p<0.001)$, Ap. caissara and Ax. corrugata, Ap. caissara and D. reticulatum, and Ap. caissara and seawater $(p<0.01)$ (Supplementary Table S3).

Using the Shannon diversity index, the highest and lowest values were observed in sediment and seawater, respectively (Table 1). There was a significant difference among the categories according to ANOVA; this was registered between sediment and each sponge species, $D$. reticulatum and seawater, seawater and sediment $(p<0.001)$, Ap. caissara and Ax. corrugata, Ax. corrugata and D. reticulatum, and Ap. caissara and seawater $(p<0.01)$ (Supplementary Table S3). The sediment again showed the highest Simpson diversity index, followed by Ap. caissara, D. reticulatum, Ax. corrugata, and seawater (Table 1). According to ANOVA, the categories were significantly different and this was registered between Ap. caissara and Ax. corrugata, D. reticulatum and Ax. corrugata, seawater and Ap. caissara, seawater and D. reticulatum, Ax. corrugata and sediment, and seawater and sediment (Supplementary Table S3). 


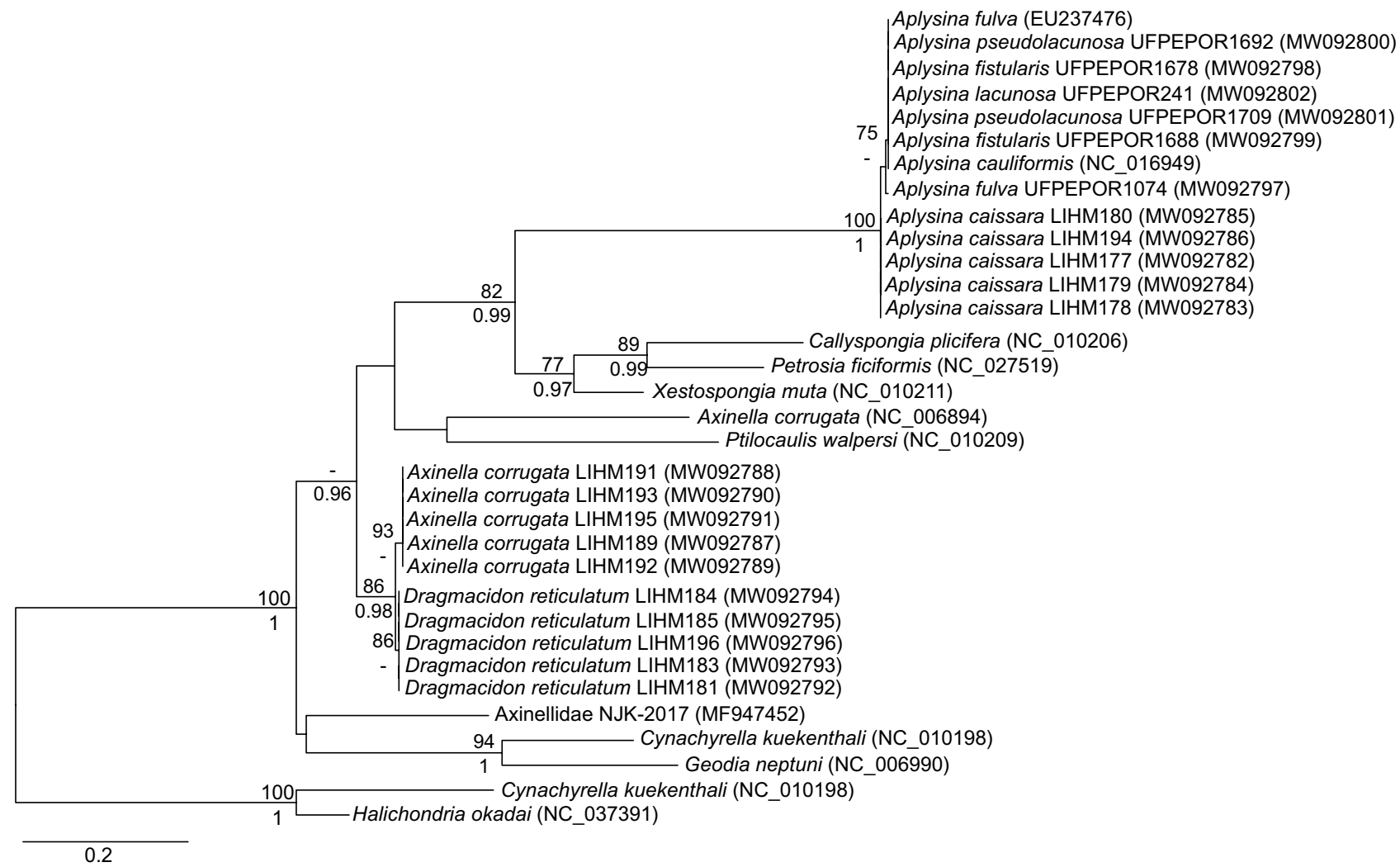

Figure 1. Phylogenetic inference of the Axinellidae and Aplysinidae families based on the cytochrome $b$. The Maximum Likelihood tree (-ln likelihood: -2655.123092) is shown. ML bootstrap values (>75\%) and Bayesian posterior probabilities $(>0.95)$ are shown above and below branches, respectively.

\begin{tabular}{|r|c|c|c|c|c|}
\hline $\begin{array}{l}\text { Samples (index } \pm \text { standard deviation } \\
(\text { SD }))\end{array}$ & Ac & Ax & Dr & SW & SD \\
\hline Sobs \pm SD & $2763.2 \pm 101.11$ & $2331.8 \pm 224.69$ & $2312.8 \pm 619.24$ & $2848.4 \pm 76.41$ & $10,886.6 \pm 189.5$ \\
\hline Chao \pm SD & $5106.27 \pm 221.91$ & $4200.5 \pm 442.6$ & $3822.98 \pm 341.84$ & $6158.08 \pm 326.54$ & $17,048.26 \pm 345.12$ \\
\hline ACE \pm SD & $5710.87 \pm 349.0$ & $4457.02 \pm 511.94$ & $4175.33 \pm 183.33$ & $6952.41 \pm 399.28$ & $18,513.73 \pm 462.99$ \\
\hline Shannon \pm SD & $4.68 \pm 0.021$ & $3.85 \pm 0.332$ & $4.95 \pm 0.67$ & $3.54 \pm 0.076$ & $7.46 \pm 0.032$ \\
\hline Inverse Simpson \pm SD & $0.96 \pm 0.003$ & $0.87 \pm 0.037$ & $0.96 \pm 0.033$ & $0.86 \pm 0.007$ & $0.99 \pm 0.00024$ \\
\hline Pielou's evenness \pm SD & $0.60 \pm 0.005$ & $0.50 \pm 0.04$ & $0.64 \pm 0.07$ & $0.44 \pm 0.008$ & $0.80 \pm 0.002$ \\
\hline
\end{tabular}

Table 1. Values for richness, diversity and evenness indices. Ac: Aplysina caissara, Ax: Axinella corrugata, Dr: Dragmacidon reticulatum, SW: seawater, SD: sediment, Sobs: estimated richness.

The Pielou's evenness index was the highest for sediment and the lowest for seawater (Table 1). The samples were significantly different according to ANOVA and this was observed between the categories, sediment and each sponge species, and between Ap. caissara and seawater, D. reticulatum and seawater, Ax. corrugata and D. reticulatum, seawater and sediment $(p<0.001)$, and Ap. caissara and Ax. corrugata $(p<0.01)$ (Supplementary Table S3).

Prokaryotic community composition. In total, 64 prokaryotic phyla were detected among all samples (Fig. 2a, Supplementary Table S4a). Sediment samples comprised the most diverse community with 59 phyla, followed by D. reticulatum, seawater, Ax. corrugata, and Ap. caissara with 49, 47, 41, and 40 phyla, respectively (Supplementary Table S4a). Overall, the most abundant phyla were Proteobacteria (average relative abundance of $30.50 \%$ ), Bacteria (unclassified, 13.55\%), Cyanobacteria (11.48\%), Crenarchaeota (9.41\%), and Chloroflexi (6.21\%) (Fig. 2a, Tables S4a, S5a). Chloroflexi (26.54\%), Crenarchaeota (18.56\%), and Proteobacteria (13.40\%) were the most abundant phyla associated with Ap. caissara (Fig. 2a, Tables S4b, S5a). For Ax. corrugata, the communities were dominated by Bacteria (unclassified, 45.03\%), Proteobacteria (23.18\%), and Cyanobacteria (10.37\%) (Fig. 2a, Tables S4c, S5a). The most abundant phyla associated with D. reticulatum were Proteobacteria (38.52\%), Cyanobacteria (10.9\%), and Firmicutes (10.42\%) (Fig. 2a, Tables S4d, S5a). The most abundant phyla detected in seawater were Proteobacteria (47.2\%) and Cyanobacteria (35.6\%) (Fig. 2a, Tables S4e, S5a). 

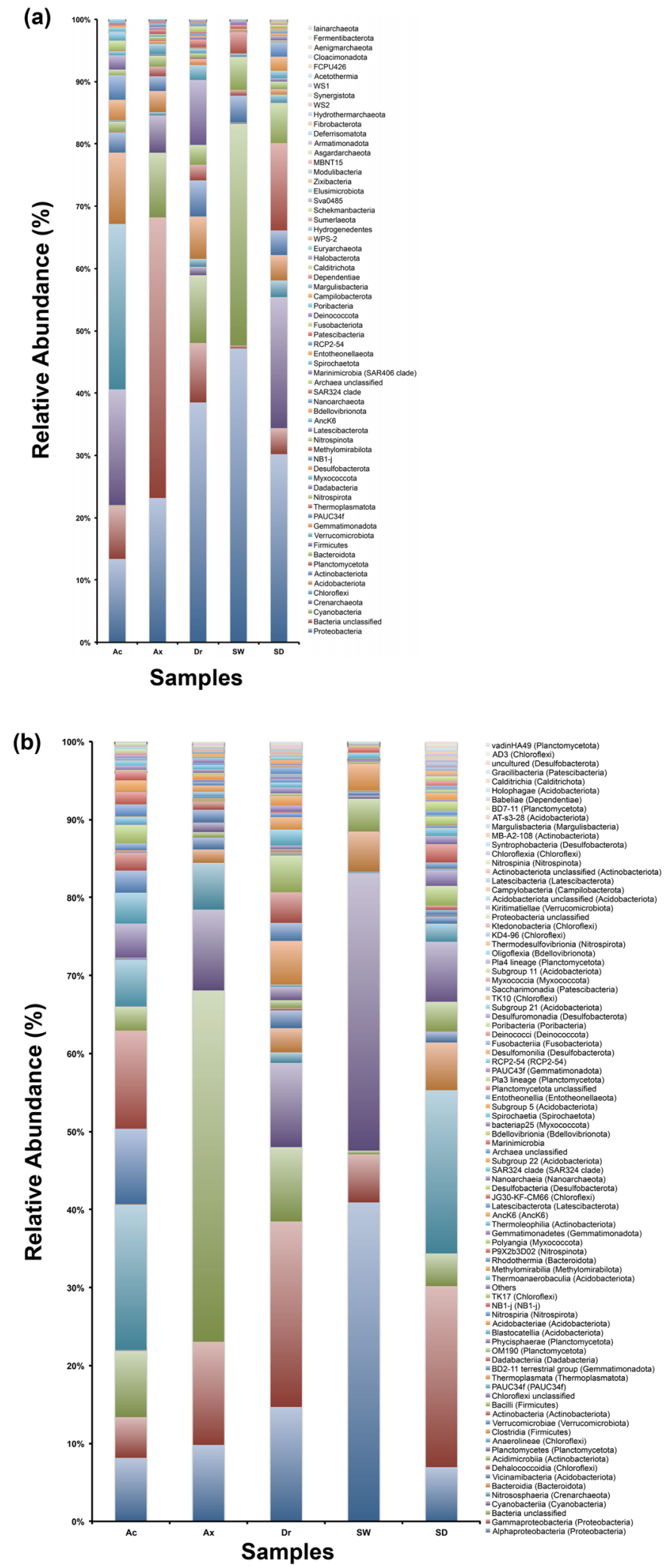

Figure 2. Phylum-(a) and class-level (b) prokaryotic community composition in marine sponges, seawater, and sediment. Compositional data for Ap. caissara (Ac), Ax. corrugata (Ax), D. reticulatum (Dr), seawater (SW), and sediment $(\mathrm{SD})$ are shown. Results obtained using pooled replicate samples $(\mathrm{n}=5)$ within each sample category are displayed. (a) All 64 prokaryotic phyla are presented. (b) Classes with relative abundance $>0.01 \%$ are shown, while relative abundance $<0.01 \%$ are grouped within others ( 88 classes). 
Sediment was dominated by Proteobacteria (30.21\%), Crenarchaeota (21.04\%), and Planctomycetota (14\%) (Fig. 2a, Tables S4f., S5a). Acidobacteriota, AncK6, Chloroflexi, Dadabacteria, Gemmatimonadota, Nitrospinota, PAUC34f, Poribacteria, and Spirochaetota were statistically $(p<0.05)$ more abundant in Ap. caissara when compared to all other categories. Additionally, the phyla Crenarchaeota, Deinococcota, and Entotheonellaeota were statistically $(p<0.05)$ more enhanced in Ap. caissara as compared specifically to Ax. corrugata and D. reticulatum. Only Bacteria (unclassified) was significantly $(p<0.05)$ abundant in Ax. corrugata when compared to the other sponge species. Four phyla (Actinobacteriota, Archaea (unclassified), Firmicutes, and Fusobacteriota) were significantly $(p<0.05)$ enriched in D. reticulatum as compared to Ap. caissara and Ax. corrugata. The phyla that were significantly $(p<0.05)$ more abundant in the axinellids species than Ap. caissara were Cyanobacteria, Desulfobacterota, Marinimicrobia, Methylomirabilota, Planctomycetota, Proteobacteria, RCP2-54, SAR324, Thermoplasmatota, and Verrucomicrobiota.

Overall, 169 classes of prokaryotes were retrieved (Fig. 2b, Supplementary Table S4b). The most diverse community composition was observed in the sediment with 151 classes, followed by D. reticulatum, seawater, Ax. corrugata, and Ap. caissara with 126, 113, 105, and 95 classes, respectively (Supplementary Table S4b). The most dominant classes were Alphaproteobacteria (16.1\%), Gammaproteobacteria (14.3\%), Bacteria (unclassified, 13.54\%), and Cyanobacteriia (11.38\%), (Fig. 2b, Supplementary Tables S4a, S5b). For Ap. caissara, the most abundant classes were Nitrososphaeria (18.56\%) and Dehalococcoidia (12.58\%) (Fig. 2b, Tables S4b, S5b). The most dominant classes associated with Ax. corrugata were Bacteria (unclassified, $45.03 \%$ ), followed by Gammaproteobacteria (13.25\%), and Cyanobacteriia (10.37\%) (Fig. 2b, Tables S4c, S5b). The classes Gammaproteobacteria (23.77\%), Alphaproteobacteria (14.7\%), and Cyanobacteriia (10.8\%) were most abundantly associated with $D$. reticulatum (Fig. 2, Tables S4d, S5b). For seawater, the dominant classes were Alphaproteobacteria (41\%) and Cyanobacteriia (35.6\%) (Fig. 2b, Tables S4e, S5b). The classes Gammaproteobacteria (23.24\%) and Nitrososphaeria (21\%) were abundantly detected in sediment (Fig. 2b, Tables S4f., S5b). Anaerolineae, Dehalococcoidia, and Vicinamibacteria were significantly $(p<0.05)$ enhanced in Ap. caissara compared to all other categories. These classes, along with Acidimicrobiia and Nitrososphaeria, were significantly $(p<0.05)$ abundant in Ap. caissara when compared to axinellids. In contrast, Actinobacteria, Bacilli, Bacteroidia, Cyanobacteria, Planctomycetes, and Verrucomicrobiae (Verrucomicrobiota) were significantly $(p<0.05)$ enhanced in axinellids when compared to Ap. caissara. For Ax. corrugata, only Bacteria (unclassified) was significantly $(p<0.05)$ abundant when compared to the other sponge species.

To obtain insights regarding the unclassified lineages associated with Ap. caissara, Ax. corrugata, and D. reticulatum, further analyses were performed at the operational taxonomic unit (OTU) level. Overall, from a total of 19,978 OTUs assigned to sponge species, 10,796 were unclassified at certain level of taxonomic affiliation (i.e. from phylum to genus). The NCBI Type Strain database revealed that $89.24 \%$ of these previously unclassified OTUs had similarity below $97 \%$. Among them, $0.86 \%$ and $2.43 \%$ of Proteobacteria and Bacteroidota, respectively, have been detected in other sponge species (Table S6a-b). Furthermore, 40 unclassified OTUs did not fulfil our BLASTn requirements with no identified match against the NCBI Type Strain database (Table S6b). However, the Silva database indicated that $63.58 \%$ of the unclassified OTUs had similarity below 97\%. Among them, 92.3, 68.2, 66.6, 58.2, and 29.96\% of Crenarchaeota, Chloroflexi, Spirochaetota, Bacteria (unclassified), and Proteobacteria, respectively, have been identified in other sponge species (Table S6c-d).

Specificities and commonalities: shared and exclusive OTUs. The Venn diagram revealed that the most OTUs $(n=37,570)$ were specific for each sample category and a few (162 OTUs) were shared among them (Fig. 3). For Ap. caissara, $79.55 \%$ of the communities were specific to this host species and the most abundant OTUs were affiliated to Bacteria (unclassified, 1147 OTUs in 6761 sequences), Dehalococcoidia (544 OTUs in 4413 sequences), Alphaproteobacteria (486 OTUs in 2227 sequences), and Vicinamibacteria (418 OTUs in 2055 sequences) (Supplementary Table S7a). For Ax. corrugata, $56.6 \%$ of the communities were specific to this species and the most dominant OTUs were affiliated to Bacteria (unclassified, 1471 OTUs in 12,979 sequences), Gammaproteobacteria (625 OTUs in 5094 sequences), and Alphaproteobacteria (404 OTUs in 6660 sequences) (Supplementary Table S7b). For D. reticulatum, 57.8\% of the communities were specific to this host and the most dominant OTUs were affiliated to Gammaproteobacteria (1012 OTUs in 11,785 sequences), Bacteria (unclassified, 597 OTUs in 4723 sequences), Alphaproteobacteria (504 OTUs in 7422 sequences), and Clostridia (Firmicutes, 500 OTUs in 8514 sequences) (Supplementary Table S7c).

In the category of environmental samples, $43.24 \%$ of the communities were specifically detected in seawater and the dominant OTUs were affiliated to Alphaproteobacteria (1211 OTUs in 3203 sequences) and Cyanobacteriia (518 OTUs in 1156 sequences) (Supplementary Table S7d). For sediment, 87\% of the communities were specific to it and the most dominant OTUs were affiliated to Gammaproteobacteria (3253 OTUs in 37,716 sequences), Bacteria (unclassified, 2407 OTUs in 12,758 sequences), Planctomycetes (1532 OTUs in 15,522 sequences), Nitrososphaeria (1263 OTUs in 11,283 sequences), Bacteroidia (1219 OTUs in 10,729 sequences), and Alphaproteobacteria (1087 OTUs in 9734 sequences) (Table S7e).

The core (composed of OTUs present in all categories, but not in all replicates) corresponded to $0.36 \%$ of the communities present in all categories (Fig. 3), and contained most sequences (42.12\%, Supplementary Table S7f.). Among them, the most abundant OTUs were affiliated to Alphaproteobacteria (33 OTUs in 175,269 sequences), Gammaproteobacteria (28 OTUs in 36,947 sequences), Bacteroidia (20 OTUs in 19,305 sequences), Cyanobacteriia (10 OTUs in 188,769 sequences), and Planctomycetes (nine OTUs in 5732 sequences) (Supplementary Table S7f).

Linear discriminant analysis effect size (LEfSe) analysis. Fifty-four taxonomic affiliations with a phylum-class-levels categorisation had the linear discriminant analysis (LDA) score above two, which explained 


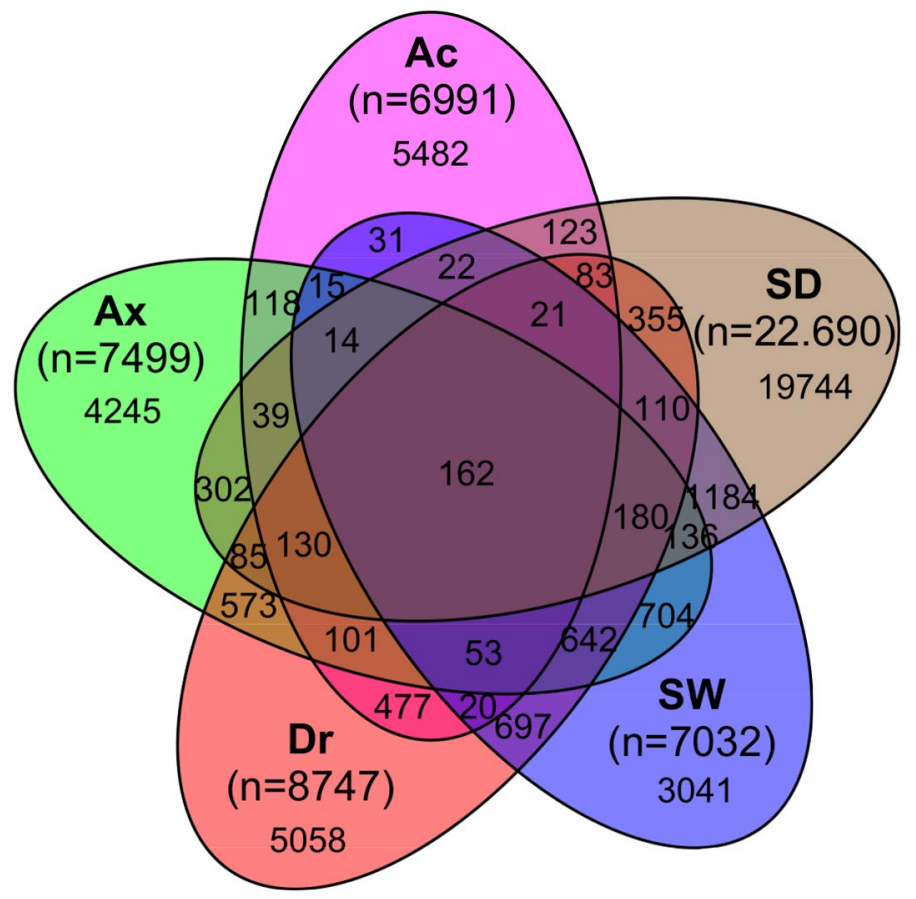

Figure 3. Venn diagram. All OTUs detected in Ap. caissara (Ac, magenta), Ax. corrugata (Ax, green), D. reticulatum (Dr, red), seawater ( $\mathrm{SW}$, blue), and sediment (SD, brown).

the variability observed in the prokaryotic communities associated with sponge species and detected in environmental samples. Sediment had 18 lineages, followed by Ap. caissara, D. reticulatum, seawater, and Ax. corrugata with 16, 12, seven, and one, respectively (Supplementary Table S8). The lineages are presented in the order of higher to lower LDA scores. For Ap. caissara, the lineages were affiliated to Dehalococcoidia, Chloroflexi, PAUC34f, BD2_11, TK17, Dadabacteriia, Dadabacteria, P9X2b3D02, Nitrospinota, Rhodothermia, JG30_KF_ CM66, bacteriap25, PAUC43f, Subgroup_11, Deinococcota, and Deinococci (Fig. 4, Supplementary Table S8). In the case of Ax. corrugata, the only lineage with LDA score above two was Bacteria (unclassified) (Fig. 4, Supplementary Table S8). For D. reticulatum, lineages were affiliated to Gemmatimonadetes, Subgroup_5, RCP2_54, Elusimicrobiota, Desulfobacterota (uncultured), Planctomycetes, Parcubacteria, Nanoarchaeota, Nanoarchaeia, MBA_A2_108, Acidobacteriota, and TK10 (Fig. 4, Supplementary Table S8). In seawater, the bacterial lineages were Marinimicrobia, SAR324, Bdellovibrionia, Margulisbacteria, Proteobacteria (unclassified), WPS_2, and Desulfobacterota (Fig. 4, Supplementary Table S8). In sediment, the significant prokaryotic lineages were OM190, NB1_j, Nitrospirota, Subgroup_22, Nitrospiria, Plactomycetota, Pla3_lineage, Latescibacterota, Pla4_ lineage, MBNT15, Syntrophobacteria, Thermodesulfovibrionia, Subgroup_21, KD4_96, Alphaproteobacteria, Myxococcota, Crenarchaeota, and Bacteroidia (Fig. 4, Supplementary Table S8).

Ordination of prokaryotic OTUs. The Bray-Curtis dissimilarity used in non-metric multidimensional scaling (nMDS) revealed three main groups with all replicates from: (i) Ap. caissara, (ii) sediment, and (iii) Ax. corrugata, D. reticulatum, and seawater (Fig. 5a). Replicates from Ap. caissara, seawater, and sediment were grouped together, whereas a more dissimilar pattern was observed among replicates from Ax. corrugata and D. reticulatum (Fig. 5a). The differences observed in Bray-Curtis dissimilarity among sponge species, seawater, and sediment was significant $(p<0.001)$ as confirmed by ADONIS.

Functional prediction. The genomes included in this analysis encompassed one archaeal and four bacterial phyla for sponges, one archaeal and eight bacterial phyla for seawater and, three archaeal and four bacterial phyla for sediment (Supplementary Table S1b-d). In marine sponge, 25 genomes were affiliated to Streptomyces and 17 to Rhodobacteraceae (Supplementary Table S1b). In seawater, the most abundant genomes were affiliated to Vibrionaceae $(n=199)$, Rhodobacteraceae $(n=128)$, Flavobacteriaceae $(n=81)$, and Pseudoalteromonadaceae $(n=45)$ (Supplementary Table S1c). In sediment, the dominant genomes were affiliated to Flavobacteriaceae $(n=32)$, Rhodobacteraceae $(n=24)$, Vibrionaceae $(n=19)$, and Streptomyces $(n=12)$ (Supplementary Table S1d).

In total, 9493 Kyoto Encyclopedia of Genes and Genomes (KEGG) Orthologs (KOs) were predicted to be present among sponge species, seawater, and sediment (Supplementary Table S9). The nMDS constructed with the relative abundance of KOs revealed a similar pattern of distribution as observed in the 16S rRNA gene; all replicates from the categories Ap. caissara, seawater, and sediment were each grouped together, whereas replicates from $A x$. corrugata and D. reticulatum were more dissimilar and clustered together (Fig. 5b). ADONIS revealed 


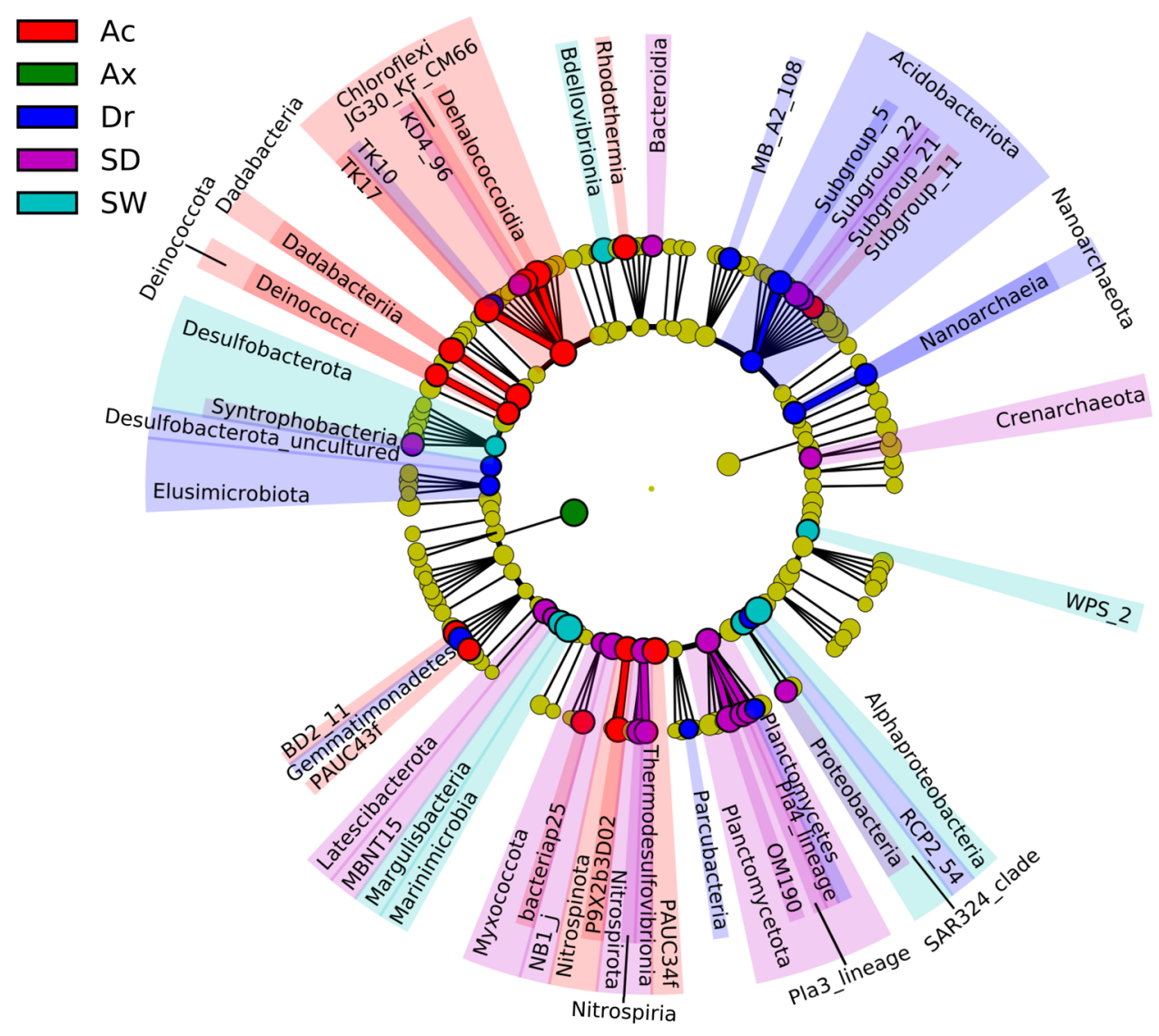

Figure 4. The linear discriminant analysis (LDA) effect size (LEFSe) analysis. Taxonomic representation of statistically and biologically consistent differences among Ap. caissara (Ac), Ax. corrugata (Ax), D. reticulatum (Dr), seawater (SW), and sediment (SD).

that the observed differences in the functional predictions among sponge species, seawater, and sediment were significant $(p<0.001)$.

In total, 392 enriched pathways were detected; according to LEfSe analysis, 253 were significantly distinct among categories. These were distributed as follows: Ap. caissara with 104 significantly $(p<0.05)$ distinct predicted pathways, followed by $D$. reticulatum, seawater, Ax. corrugata, and sediment with $60,59,16$, and 14 distinct pathways, respectively (Supplementary Table S10). In Ap. caissara the most abundant predicted pathways were related to $(i)$ biosynthesis of secondary metabolites (e.g. clavulanic biosynthesis, flavonoid biosynthesis, isquinoline alkaloid biosynthesis, and stilbenoid, diarylheptanoid, and gingerol biosynthesis), (ii) viral infectious diseases (e.g. hepatitis B and C, herpes simplex infection, and influenza A), (iii) xenobiotics biodegradations and metabolism (e.g. ethylbenzene degradation, naphthalene degradation, nitrotoluene degradation, polycyclic aromatic hydrocarbon degradation), (iv) energy metabolism pathways (i.e. carbon fixation pathways in prokaryotes, methane, nitrogen, oxidative phosphorylation, and sulphur metabolism), and (v) metabolism of terpenoids and polyketides (i.e. biosynthesis of ansamycins, biosynthesis of vancomycin group antibiotics, polyketide sugar unit biosynthesis, terpenoid backbone biosynthesis) (Supplementary Table S10). For Ax. corrugata, predicted pathways were registered in: (i) xenobiotics biodegradation and metabolism (i.e. chlorocyclohexane and chlorobenzene degradation, drug metabolism - cytochrome P450, drug metabolism-other enzymes, and metabolism of xenobiotics by cytochrome P450); (ii) biosynthesis of other secondary metabolites (i.e. neomycin, kanamycin and gentamicin biosynthesis, and penicillin and cephalosporin biosynthesis), and (iii) metabolism of terpenoids and polyketides (i.e. biosynthesis of enediyne antibiotics) (Supplementary Table S10). For D. reticulatum, the dominant predicted pathways were recorded for $(\boldsymbol{i})$ xenobiotics biodegradation and metabolism (e.g. atrazine degradation, chloroalkane and chloroalkene degradation, aminobenzoate degradation, toluene degradation, xylene degradation) and (ii) metabolism of terpenoids and polyketides (e.g. biosynthesis of siderophore group nonribosomal peptides, biosynthesis of type II polyketide products, nonribosomal peptide structures, and type I polyketide structures) (Supplementary Table S10).

\section{Discussion}

To the best of our knowledge, this is the first survey addressing the prokaryotic community composition, diversity, specificity, and putative functionality of the sympatric southwestern Atlantic sponges Ap. caissara, Ax. corrugata, and D. reticulatum. 
(a)

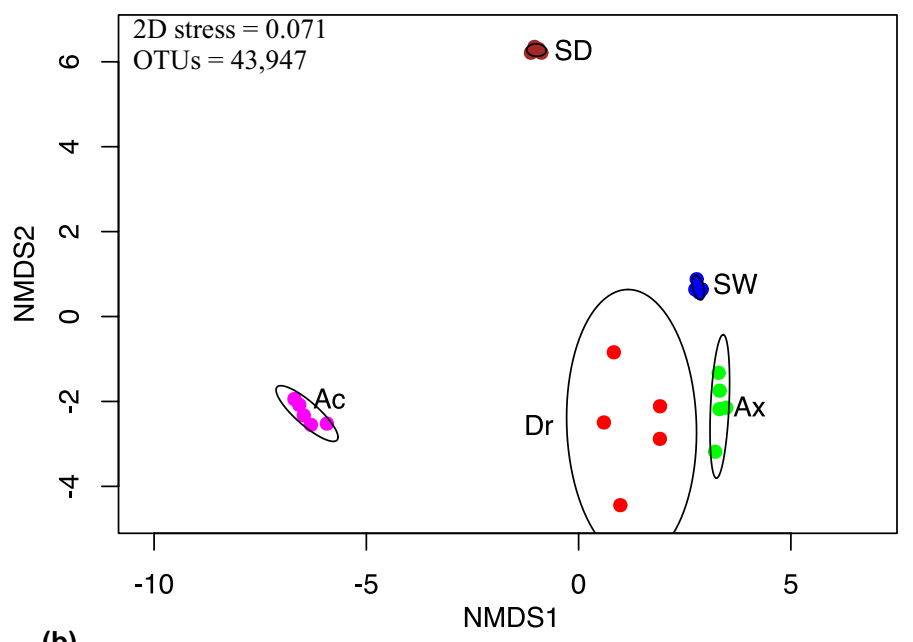

(b)

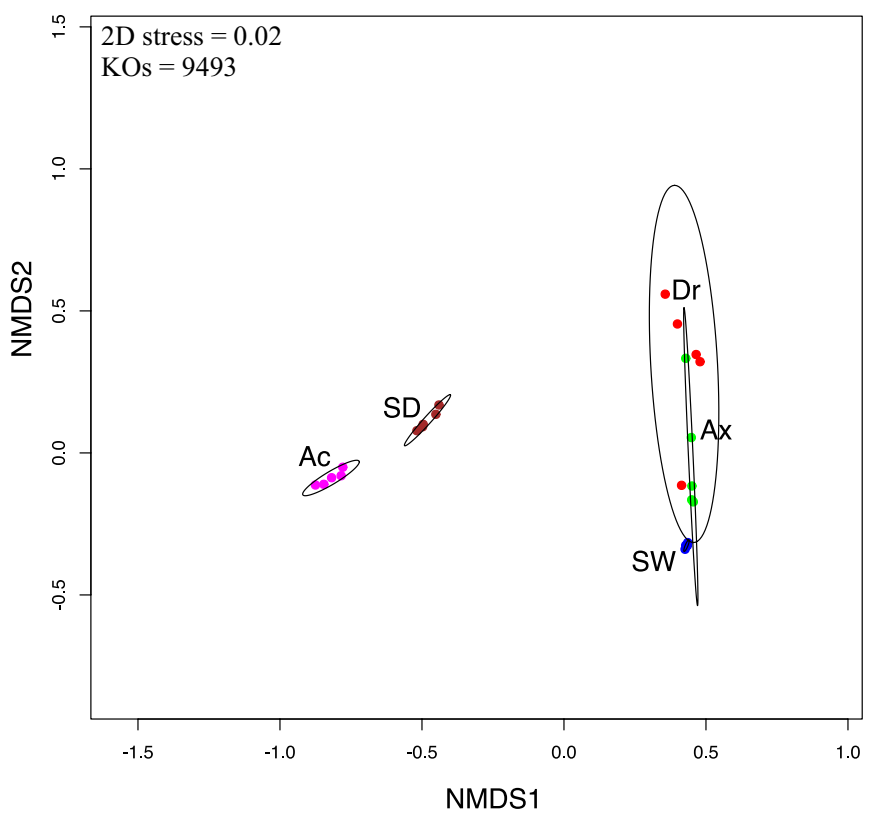

Figure 5. Non-metric multidimensional scaling (nMDS) plots. (a) nMDS based on Bray-Curtis distances calculated from the normalized 97\% OTU table and (b) predicted KEGG Orthologs for each sponge species, seawater, and sediment. Ap. caissara (Ac), Ax. corrugata (Ax), D. reticulatum (Dr), seawater (SW), and sediment (SD).

In an attempt to overcome the difficulty encountered in the traditional taxonomy, DNA barcoding was implemented to assist in the identification ${ }^{7}$. Due to the gene organisation not being conserved at the poriferan mitogenomes and the presence of certain linear ones ${ }^{25}$, it was difficult to amplify the standard barcoding fragment for eukaryotic animals, the mitochondrial gene for the subunit I of the cytochrome c oxidase (cox-1). Different markers were tested: a downstream region (I3M11) of cox- $1^{26}$, internal transcribed spacer (ITS) ${ }^{27}$, and a short LSU rRNA fragment ${ }^{28}$. Only recently ${ }^{28}$, the C2-D2 region of $28 \mathrm{~S}$ rDNA was proven to be universally suitable for barcoding all sponge classes, providing high resolution and easy amplification. The ITS could also provide resolution in the case of Aplysina species ${ }^{29}$, whereas, cox-1 was unable to separate species within the family Aplysinidae ${ }^{30}$.The cob gene is evidently capable of differentiating sponge species ${ }^{14}$; therefore, the cob gene provided robust separation between Ax. corrugata and D. reticulatum, and it could separate Ap. caissara from all other Aplysina species. However, cob was unable to differentiate the other five Aplysina species and care should still be taken when barcoding the family Aplysinidae. There is only one cob sequence for Ax. corrugata (NC006894) available at the GenBank; however, it showed less than 83\% sequence similarity to our sequence and was grouped outside the Ax. corrugata and D. reticulatum cluster. Hence, with a more comprehensive sampling, the cob gene may help improve the molecular identification of these sponge species; although, it is outside the scope of the present contribution. 
To summarise the differences in the prokaryotic structure associated with each sponge species and those found in the environmental samples, the alpha diversity indices were compared. Recently, the surrounding sediment has more often been included in the analysis but not as frequently as seawater ${ }^{10,31,32}$. Sediment presented the highest values for richness, diversity, and evenness indices. Furthermore, sediment did not approach a plateau at the rarefaction curve, which was observed in other studies ${ }^{10,31,32}$. Surprisingly, seawater reached the plateau, even though it presented the second highest values for observed richness investigated in this survey. Independently of the high-throughput sequence platform used, seawater is typically asymptote ${ }^{10,32-34}$, whereas it might approach the plateau when a rarefied dataset is used ${ }^{31}$. It was observed that the prokaryotic richness in most of the sponges exhibited a less complex community than those found in seawater and sediment ${ }^{10}$. Similarly, $A p$. caissara, Ax. corrugata, and D. reticulatum presented lower observed richness than the ones registered in sediment, whereas Ap. caissara and D. reticulatum may approach the richness registered for seawater. In contrast, for diversity and evenness indices, the sponge species had values in-between sediment and seawater. Cleary and colleagues $^{31}$ detected a correlation between evenness and richness; biotypes with high evenness also showed the highest richness. In the present study, a shift was detected between Ap. caissara and D. reticulatum, where the former had higher richness and lower evenness compared to the latter. The evenness might have played an essential role by $(\boldsymbol{i})$ regulating the community resistance to invasion, $(\boldsymbol{i i})$ maintaining the functional stability of an ecosystem, and (iii) responding to the effect of an unexpected selective stress ${ }^{35,36}$. The high evenness registered from sponge species indicates that these species would be able to overcome invasion and stress while retaining the functionality of their communities.

In the present study, 40-47 distinct prokaryotic phyla were associated with three sponge species, which was within the range of 41-72 detected in sponges worldwide ${ }^{10,12}$. Regardless of the technique used, the most dominant prokaryotic phyla associated with sponges were affiliated to Proteobacteria, Chloroflexi, Cyanobacteriota, Acidobacteriota, and Actinobacteriota ${ }^{10,31,32,37}$. In the present survey, these were also the most dominant, along with Bacteria (unclassified) and Crenarchaeota. The most dominant prokaryotic communities associated with other Aplysina collected in seven sampling places (A. aerophoba, A. cavernicola, A. fulva, A. archeri, and A. cauliformis) were Proteobacteria (relative abundance of $\sim 15 \%)$, unclassified $(\sim 12 \%)$, Chloroflexi $(\sim 15 \%)$, Acidobacteriota $(\sim 8 \%)$, and Actinobacteriota $(\sim 8 \%)$, which were distinct from the ones detected for Ap. caissara in the present study in terms of associated-phylum and relative abundances ${ }^{10}$. Likewise, for Ax. corrugata collected at the Bahamas and United States, the most dominant prokaryotic phyla were Proteobacteria $(\sim 15 \%)$, Crenarchaeota $(\sim 12 \%)$, unclassified $(\sim 5 \%)$, and Cyanobacteriota $(\sim 5 \%)^{10}$, which were again different from the ones associated with the Brazilian Ax. corrugata. Using a cloning library, seven phyla were detected associated with $D$. reticulatum collected from the north coast of São Paulo, in which the most dominant were Cyanobacteria and Proteobacteria ${ }^{38}$, contrasting with the results obtained in the present survey. Certain studies demonstrated that the most abundant classes associated with several sponges collected worldwide, including five Aplysina species and Ax. corrugata, were Gammaproteobacteria and Alphaproteobacteria ${ }^{10,32}$, whereas in the present survey, the most abundant were Bacteria (unclassified), Gammaproteobacteria, Alphaproteobacteria, Nitrososphaeria, and Cyanobacteriia. Furthermore, the prokaryotic communities associated with the three sponge species were markedly different from the ones obtained from seawater and sediment. Based on the differences in the relative abundance and community compositions associated with the sympatric species Ap. caissara, Ax. corrugata, and D. reticulatum, the host seemingly plays a deterministic role in shaping the structure of their own prokaryotic communities. These complex sponge-prokaryotic associations are a defined pattern with robust supportive evidence from numerous sponge species inhabiting the environmentally diverse marine habitats and climate zones $^{10,33,39,40}$. The differences that encompassed three sympatric sponge species, of which two were phylogenetically related, might also be correlated to the evolutionary history between the symbionts and the sponge species. The unclassified OTUs associated with Ap. caissara, Ax. corrugata, and D. reticulatum further confirmed that the Brazilian sponge species represent a reservoir of novel prokaryotic diversity.

In addition to the assessment of the prokaryotic community composition and diversity, an attempt was made to correlate the capability of performance by the prokaryotic lineages enhanced in each sponge species, and their corresponding predicted functions registered. Notably, the functional prediction based on the 16S rRNA gene must be validated by metagenomics or whole genome sequencing. However, our approach was adequate, as it allowed the inclusion of prokaryotic genomes of sponges, seawater, and sediment to the dataset provided by the software, although a bias was observed within the taxonomic affiliation of the genomes. The majority of genomes in marine sponges were affiliated with Actinobacteria and Proteobacteria, which are well known to contain several secondary metabolites, whereas Streptomyces accounted for $30 \%$ of the bacteria isolated from marine sponge capable of producing antimicrobial compounds ${ }^{41}$. A significant effort has been made to sequence bacterial genomes that exhibit biotechnological potential, demonstrating a huge gap in the knowledge regarding the capability of the bacteria associated with marine sponges. Even with this bias, the approach provided insights into the potential functional aspects of the prokaryotic communities associated with three Brazilian sponge species. As described below, several correlations could be made between prokaryotic members and predicted functions, whereas many lineage(s) capable of performing them are yet to be discovered.

Among all the prokaryotic phyla and class levels in this survey, 54 were responsible for the variability detected among categories. The phyla enhanced in Ap. caissara have been associated with other marine sponges, including several Aplysina species, with relative abundances ranging from 0.001 to approximately $32 \%$. Besides, some of these phyla contained several sponge-enriched clusters ${ }^{9,42-44}$. Metagenome-assembled genomes (MAGs) obtained from Ircinia ramosa demonstrated that Chloroflexi and Dadabacteria participated in nitrogen and sulphur cycling. In contrast, Nitrospinota were involved in sulphur cycling, and these three phyla were capable of synthesising distinct B-vitamins ${ }^{45}$. Furthermore, Chloroflexi associated with Ap. aerophoba showed features for glycolysis, carbon fixation, machinery for transcription, purine and pyrimidine metabolism, biosynthesis of most amino acids and cofactors, and potential aromatic degradation ${ }^{46}$, as well as halogenases involved in 
the production of brominated compound ${ }^{47}$. Additionally, a Chloroflexi bacterium was the likely producer of a nonribosomal peptide synthase (NRPS) ${ }^{48}$. Several functional capabilities were detected in the genomes of marine Dadabacteria, Nitrospinota, and Deinococcota ${ }^{49-54}$, which were enhanced in A. caissara; however, further research is required to corroborate their performance in the sponge. Metabolic reconstruction of the metagenome of Ap. aerophoba and Petrosia ficiformis revealed that PAUC34f members contained genes $(i)$ involved in glycolysis and oxidative phosphorylation, (ii) encoding numerous enzymes involved in the uptake and/or metabolism of nitrogen and sulphate and in the production of amino acids, vitamins, purines and pyrimidines, and (iii) encoding polyketides (PKS) modules and proteins, and several secondary metabolites biosynthesis gene clusters ${ }^{55}$. Several of the classes enriched in Ap. caissara have been associated with sponge species ${ }^{46,56}$. Among them, the best characterised is Dehalococcoidia, detected in numerous sponges including several Aplysina species, but with relative abundances of only $0.25 \%{ }^{46,56}$. Marine Dehalococcoidia has been shown to be involved in the biogeochemical cycling of carbon, methane, and sulphur, and in bioremediation, especially of the chloroorganic pollutants. Aplysina species are well known for their halogenated substances, including the brominated compounds, which might be used by Dehalococcoidia. Hence, it may play an essential role in disarming the chemical defence systems and in attenuating signal agents by dehalogenating the halogenated signalling molecules ${ }^{57-61}$. To the best of our knowledge, genome and functional information are unavailable for members of the other classes enhanced in $A p$. caissara; thus, no inference can be derived at present. Overall, the features described above were predicted in Ap. caissara and it is tempting to speculate that they might be performed by the lineages enriched in this sponge host. For instance, Chloroflexi, PAUC34f, and Deinococcota might be responsible for the metabolism of terpenoids and PKS and the biosynthesis of other secondary compounds, whereas metabolism of amino acid, cofactors, and vitamins might be performed by all phyla, except Dadabacteria. Additionally, it is tempting to speculate that Dehalococcoidia might be responsible for the energy metabolism and xenobiotics biodegradation predicted in Ap. caissara. Furthermore, as several members were involved in the biogeochemical cycling of nitrogen, sulphur, and carbon, it might indicate that functional redundancy is occurring in this host. To provide further support to the lineages that might be involved in the predicted functions enriched in Ap. caissara and their specificity, these taxonomic levels comprised 3202 OTUs detected in Ap. caissara, from which 2931 were host specific.

The class TK-10, enriched in D. reticulatum, was the producer of halogenases in several Aplysina species an essential enzyme in the biosynthesis of brominated and chlorinated secondary metabolites ${ }^{58}$. Dragmacidins are bromoindole alkaloid that inhibit the growth of several cancer cell lines and present antimicrobial and antiinflammatory activities ${ }^{62,63}$. Thus, it can be considered that TK-10 are involved in the metabolism of terpenoids and PKS as predicted in D. reticulatum. The class Planctomycetes enriched in D. reticulatum was until recently classified as a phyla ${ }^{64}$. Planctomycetes are enriched with the capacity to synthesise secondary metabolites (e.g. PKS and NRPS genes, and antimicrobial and anticancer compounds) and gene clusters responsible for biofilm formation and quorum sensing ${ }^{65,66}$. Moreover, Planctomycetes are also able to degrade herbicides ${ }^{66}$. Thus, this phylum might be responsible for cellular community, metabolism of terpenoids and PKS, and degradation and metabolism of xenobiotics. Furthermore, the class Parcubacteria have small genomes and cell sizes, and important gaps in core metabolic potential, consistent with a symbiotic lifestyle, and they display relatively low abundance in surface seawater. The features detected in the genomes obtained from several places, including marine, were amino acids and fatty acid metabolism, membrane transport, secondary metabolites biosynthesis, and aromatic compound degradation ${ }^{67-69}$. These characteristics were predicted in D. reticulatum; thus, Parcubacteria might be involved in these activities. Analyses of certain marine Desulfobacterota genomes revealed that the major predicted clusters of orthologous groups were correlated to amino acid transport and metabolism, carbohydrate transport and metabolism, and lipid transport and metabolism ${ }^{70,71}$. These features predicted in $D$. reticulatum might be performed by Desulfobacterota, even though the unclassified members were enhanced. Nanoarchaeota have thus far been detected in extreme environments; they are obligate symbionts of other Archaea, consequently they contain reduced genomes, and lack most biosynthetic pathways and functional ATPase has not been detected ${ }^{72-75}$. Based on the abovementioned and on the fact that sponges were not collected from extreme habitats, and none of the predicted functions detected in D. reticulatum were observed in the genomes of Nanoarchaeota, seemingly this phylum is indeed symbiont of another Archaea that is associated with D. reticulatum. In this study, Nanoarchaeota was for the first time detected in marine sponges and the functional and ecological relationships among host and their associated microbiota are yet to be addressed. Furthermore, insufficient information was obtained for several lineages enhanced in D. reticulatum. These members comprised 1283 OTUs detected in D. reticulatum, from which 792 were host specific, further validating that the enriched members were likely to be performing the predicted functions.

The only member enriched in Ax. corrugata was Bacteria that could only be classified at the domain level; thus, no correlation could be drawn presently. It highlights that although our knowledge regarding the prokaryotic diversity has developed in the last decade, there is still more to be discovered. To further corroborate that this lineage is most likely carrying out the predicted functions, 1471 OTUs from a total of 1625 were host specific.

Overall, in the present study, it was demonstrated that the prokaryotic communities associated with Ap. caissara, $A x$. corrugata, and $D$. reticulatum follow a deterministic-based assembly mechanism. The sponge species play a pivotal role in selecting their own prokaryotic communities, displaying host specificity. The unclassified OTUs associated with Brazilian sponge species represented an untapped reservoir of prokaryotic diversity corroborating the hotspot ecoregions hypothesis. Although 64 prokaryotic phyla comprising 169 classes were detected, only 54 lineages were responsible for the variability detected among categories. Surprisingly, the lineages associated with three sponge species could be correlated with the predicted functions enriched in each host. Although the predictive nature of the functional profiling based on 16S rRNA marker is not a substitute for the whole metagenome profiling in microbial ecology surveys, it provides a simple and cheap assessment of putative functions in the community. 


\section{Material and methods}

Sponges and environmental sampling. Samples were collected at the southern rock shores of Guaecá-

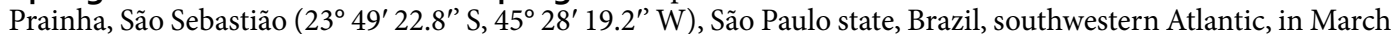
2019. Measurements of salinity and temperature at the time of sampling were $33.4 \mathrm{ppm}$ and $29.4^{\circ} \mathrm{C}$, respectively. Five individuals of each species, Aplysina caissara (endemic) (Pinheiro \& Hajdu, 2001) (Demospongiae, Verongiidiia, Aplysinidae), Dragmacidon reticulatum (Ridley \& Dendy, 1886) (Demospongiae, Axinellida, Axinellidae) and Axinella corrugata (George \& Wilson, 1919) (Demospongiae, Axinellida, Axinellidae) were collected by diving at depths of 5-6.5 m and placed separately in situ in sterile ziplock bags containing natural seawater. In situ images of the specimens were taken to aid in identification. Under similar conditions, five surrounding seawater samples (1 L each, approximately $1 \mathrm{~m}$ around the vicinity of the sponge specimens) were collected in $1 \mathrm{~L}$ sterile plastic bottles, along with sediment samples that were placed in sterile ziplock bags (approximately $2 \mathrm{~kg}$ each sample). Samples were placed in cooling boxes, transported to the laboratory (c. $30 \mathrm{~min}$ ) at Center for Marine Biology of São Paulo University (CEBIMar/USP) for initial processing. Prior to the processing, the sponge specimens were rinsed with sterile artificial seawater ${ }^{76}$ to remove loosely associated organisms. Voucher samples were preserved in $70 \%$ ethanol for taxonomic identification. Pieces from the inner part of the sponge specimens were preserved in RNAlater (QIAGEN, Hilden, Germany) at $4{ }^{\circ} \mathrm{C}$ overnight and subsequently transferred to $-20^{\circ} \mathrm{C}$, and environmental samples were maintained at $-20^{\circ} \mathrm{C}$ until further use.

Sponge identification. Morphological identification of sponges was performed using standard methods: spicule and skeletal preparations followed Hajdu and colleagues ${ }^{77}$, and spongin fibres were prepared according to Pinheiro and Hajdu ${ }^{3}$. Phylogenetic inference (DNA barcoding ${ }^{7}$ ) was used in the molecular identification of species. Polymerase chain-reaction (PCR) amplifications were performed on sponge genomic DNA (see below) targeting the cytochrome b (cob) with primer pair Diplo-cob-f1m (5'-ATGTNTTNCCTTGRGGWCAA ATGTC-3 $\left.{ }^{\prime}\right)^{78}$ and Diplo-cob-r1 (5'-GGATTGAWCGTAAWATWGCRTAAGC-3') modified from Lavrov and colleagues $^{78}$. The reaction mixture $(25 \mathrm{~mL})$ contained $1.0 \mu \mathrm{L}$ of template DNA $(\sim 20 \mathrm{ng}), 2 \mathrm{X}$ buffer GoTaq (Promega, Madison, USA), $0.24 \mathrm{mM}$ of each primer. The initial cycle of $4 \mathrm{~min}$ at $94^{\circ} \mathrm{C}, 1 \mathrm{~min}$ at $47^{\circ} \mathrm{C}$ and $1 \mathrm{~min}$ at $72{ }^{\circ} \mathrm{C}$ was followed by 35 cycles of $1 \mathrm{~min}$ at $92^{\circ} \mathrm{C}, 1 \mathrm{~min}$ at $47^{\circ} \mathrm{C}$ and $1 \mathrm{~min}$ at $72^{\circ} \mathrm{C}$ for template amplification. A final extension of $6 \mathrm{~min}$ at $72^{\circ} \mathrm{C}$ was used to complete the reaction. All PCR amplifications were carried out in a C1000 Touch thermal cycler (Bio-Rad, Hercules, CA, USA). Amplicons were visualised after electrophoresis on $1 \%$ agarose gel under UV light. The PCR products of the expected size were subjected to sequencing with the chain termination method in an ABI 3500 automatic sequencer (Applied Biosystems, Foster City, CA, USA) using the forward primer. The generated sequences were quality inspected and edited with the Sequence Scanner 2 software (Applied Biosystems, Foster City, CA, USA). To investigate the capacity of the cob to separate Aplysina species, voucher representatives obtained from Coleção de Porifera from the Universidade Federal de Pernambuco (UFPE) were subjected to the same procedures and analyses as described above. The resulting sequences were submitted to the NCBI database under the accession numbers MW092782-MW092802.

Total community DNA extraction. Genomic DNA of internal sponge body (approximately $0.25 \mathrm{~g}$ ) was extracted using DNeasy PowerSoil DNA isolation kit (QIAGEN, Hilden, Germany) according to the manufacturer's protocol. Seawater samples $(1 \mathrm{~L})$ were filtered through nitrocellulose filters $(0.2 \mu \mathrm{m}$ pore-size) (Merck Millipore, Burlington, MA, USA) using a vacuum pump. The filters were cut into small pieces and directly used for DNA extraction with the DNeasy PowerSoil DNA isolation kit following the manufacturer's protocol. Sediment samples $(2 \mathrm{~kg}$ ) were mixed, sieved, and an aliquot of $0.25 \mathrm{~g}$ was used for DNA extraction as explained above.

Phylogenetic analyses. In total, 15 sequences, five from each sponge species, were used for the phylogenetic analyses. Closest relatives were searched using the megaBLAST and BLASTn algorithms of the NCBI ${ }^{79-81}$. The sequences obtained in the present study and those retrieved from NCBI were aligned with MEGA software v. $10^{82,83}$. The FASTA format file was opened using SeaView software v. $4^{84}$ and saved as PHYLIP for jModeltest and Maximum Likelihood as well as nexus for Bayesian phylogenetic analyses. The selection of the best model for the inference was performed by jModeltest v. 2.1.6 $6^{85,86}$ using CIPRES Science Gateway website v. $3.3^{87}$; the general-time reversible model $\left(\mathrm{GTR}^{88}\right)$ was selected with a discrete gamma distribution of among-site rate variation $\left(\Gamma_{4}\right)$ and a proportion of invariant sites (I). The optimal and bootstrap maximum-likelihood inferences were performed at CIPRES Science Gateway website v. $3.3^{80}$. An optimal maximum-likelihood tree was determined using RAxML 8.2.12 $2^{89}$, with 1000 replicates, each starting from a random tree, with the GTR + I + G model. Maximum-likelihood bootstrap support was ascertained with the same software and model, using 1000 replicates. A Bayesian Markov chain Monte Carlo (MCMC) analysis was also conducted using MrBayes 3.2.6 ${ }^{90,91}$, with two runs using four chains (Metropolis-coupling) with 1000 generations, and GTR + I+ G model. All other options, including priors, were default values. The 'burn-in' period before the MCMC reached stationarity was defined as $25 \%$ of the initial trees. Tree sets from the posterior distribution of the two independent runs were concatenated from the sample of trees and assumed to be randomly sampled from the posterior probability distribution and $50 \%$ majority-rule consensus tree.

165 rDNA Illumina sequencing. An aliquot of the purified genomic DNA was submitted to the Functional Genomics Center of the Luiz de Queiroz College of Agriculture (ESALQ-USP) to perform 16S rRNA gene sequencing. Briefly, the V4-region of the $16 \mathrm{~S}$ rRNA gene, targeting the prokaryotic community, was amplified with the primer pair $515 \mathrm{~F}^{92}$ and $806 \mathrm{R}^{93}$. The reaction mixture $(25 \mu \mathrm{L})$ encompassed of $2.5 \mu \mathrm{L}$ of template DNA (20 ng $\mathrm{ll}^{-1}$ ), $0.20 \mathrm{mM}$ of each primer, 2X PCRBio Ultra Mix (PCRBiosystems, Wayne, USA). The thermal cycle 
started with $3 \mathrm{~min}$ at $95^{\circ} \mathrm{C}$, followed by 30 cycles of $30 \mathrm{~s}$ at $95^{\circ} \mathrm{C}, 30 \mathrm{~s}$ at $60^{\circ} \mathrm{C}$ and $30 \mathrm{~s}$ at $72^{\circ} \mathrm{C}$. A final extension of $10 \mathrm{~min}$ at $72^{\circ} \mathrm{C}$ was applied for reaction completion. The amplicons were subjected to Illumina sequencing using MiSeq platform.

Analyses of Sequencing data. Illumina sequences were processed using Mothur v. 1.44 ${ }^{94}$. A pipeline was optimised and executed. Briefly, paired raw reads were subjected to pre-processing through quality checking. Then, the dataset was reduced to non-identical sequences to decrease computational effort. Sequences were aligned using the reference SILVA seed v. 138 database (mothur-formatted), provided by Mothur ${ }^{95,96}$. The dataset was reduced to non-redundant sequences and were pre-clustered. Then, chimeric sequences detected with $\mathrm{UCHIME}^{97}$ were removed from the dataset. The remaining sequences were phylogenetically classified. Undesirables and singletons were removed from the dataset. Sequences were assigned to OTUs classified at $97 \%$ sequence similarity. The libraries were normalised. OTUs were further classified based on the SILVA non-redundant v. 138 database (mothur-formatted $)^{95,96}$. All 16S rRNA datasets generated in this study were deposited as Sequence Read Archive in NCBI database with Bioproject ID: PRJNA665805 (SAMN1626881-SAMN16268843). For a detailed description of the pipeline used see Supplementary Material.

Calculation of community metrics. Richness (Sobs, CHAO, and ACE), diversity (Shannon and inverse Simpson) and evenness (Pielou's evenness) indicators were calculated using the R package vegan v. 2.5-6 $6^{98}$. ANOVA was performed using R package vegan $2.5-6^{98}$ to test the significant difference in the obtained mean values from each index. For ANOVA, $p$ value of $<0.001$ was considered statistically significant. The multcomp version 1.4-13 R package ${ }^{99}$ was applied for multiple comparisons of mean values with Tukey contrasts. Statistical analyses were performed for the barchart when the sum of the relative abundance from all replicates for phyla and class were $\geq 5 \%$ and $\geq 1 \%$, respectively, and were also carried out in $\mathrm{R}^{100}$. Square root transformation was used to improve the normality of the relative abundance. The Welch's test was performed, followed by a Pos-Hoc test with Holm pairwise correction because of unequal homogeneity of variance.

The Venn diagram was constructed to determine the number of OTUs specifically assigned to each category and common to all categories using the R package VennDiagram v. 1.6.20100,101, whereas the identity of OTUs was discovered using the online tool available at http://bioinformatics.psb.ugent.be/webtools/Venn/. In this study, the core OTUs were defined as OTUs present in all categories but not necessarily in all replicates.

The unclassified OTUs associated with marine sponges at certain level of the taxonomic affiliation (from phylum to genus) were further investigated. These OTUs were subjected to BLASTn ${ }^{79-81}$ using two distinct databases: (i) type strain from NCBI (available on 02/01/2021) and (ii) SILVA non-redundant v. 138. It is important to note that standard NCBI annotation was used to collect host and isolation source information when available. The scripts used are provided in the Supplementary Material.

To determine the phylogenetic lineages responsible for the differences detected in each category (i.e. sponge species, seawater, and sediment), LEfSe v $1.0^{102}$ was performed on the Galaxy web platform ${ }^{103}$ with default parameters. Distances of the samples in each category and their respective group centroids were calculated based on Bray-Curtis distances using the function vegdist from the vegan package v. 2.5-6 in $\mathrm{R}^{98,100}$. nMDS using the Bray-Curtis dissimilarities was calculated in vegan package v. 2.5-6 in $\mathrm{R}^{98,100}$. The differences in Bray-Curtis dissimilarity among categories was tested using ADONIS.

Functional predictions. To obtain insights regarding putative functions using the $16 \mathrm{~S}$ rRNA gene, the software Tax4fun 2 v. 1.1 .5 was used ${ }^{104}$. The advantage of this programme is that in addition to the reference genome database provided, genomes of interest can also be included. To this end, genomes from marine sponges, seawater, and sediment from all five oceans were searched at $\mathrm{NCBI}^{79,80}$ using genomic DNA/RNA and RefSeq datasets with the following keywords: marine sponge, seawater, or sediment combined with bacteria or archaea. Nearly complete genome sequences with at least one copy of the 16S rRNA gene were further used. Thus, 123 prokaryotic genomes isolated from sponges and 241 and 720 genomes obtained from sediment and seawater, respectively, were analysed. The details, including taxonomic affiliations, of the added genomes can be found in Supplementary Table S1a and the pipeline used is described in Supplemental Material. Two tables were generated (functional and pathway predictions). The relative abundance of $\mathrm{KO}$ was analysed with nMDS in vegan package v. 2.5-6 in $\mathrm{R}^{98,100}$. LEfSe v 1.0 was also used here to identify the KEGG pathways as significant biomarkers for each category by calculating $\operatorname{LDA}^{10251}$, as explained above. Nonetheless, the results obtained with the predictive functional profiling using $16 \mathrm{~S}$ rRNA marker gene does not substitute metagenomic profiling and functional gene annotation and may diverge. Furthermore, due to the functional overlap, some KOs were assigned to more than one pathway.

\section{Permits}

Sampling was performed under the scientific collection permits A097B99 issued by Sistema Nacional de Gestão do Patrimônio Genético e do Conhecimento Tradicional Associado, 61460-2 issued by Sistema de Autorização e Informação sobre Biodiversidade do Instituto Chico Mendes de Conservação da Biodiversidade, both from Ministry of the Environment and 260108-001.161/2013 issued by Instituto Florestal, Secretaria do Meio Ambiente do Estado de São Paulo.

\section{Data availability}

All 16S rRNA datasets generated through this study were deposited as Sequence Read Archive in NCBI database with Bioproject ID: PRJNA665805 (SAMN1626881-SAMN16268843). All cob sequences were deposited at NCBI database under Accession Numbers: MW092782-MW092802. 
Received: 1 November 2020; Accepted: 7 April 2021

Published online: 05 May 2021

\section{References}

1. Zumberge, J. A. et al. Demosponge steroid biomarker 26-methylstigmastane provides evidence for Neoproterozoic animals. Nat. Ecol. Evol. 2, 1709-1714 (2018).

2. van Soest, R. W. et al. World Porifera database. World Porifera database (2019).

3. Pinheiro, U. dos S. \& Hajdu, E. Shallow-water Aplysina Nardo (Aplysinidae, Verongida, Demospongiae) from the São Sebastião Channel and its environs (Tropical southwestern Atlantic), with the description of a new species and a literature review of other brazilian records of the genus. Rev. Bras. Zool. (2001) https://doi.org/10.1590/s0101-81752001000500012.

4. Pinheiro, U. S., Hajdu, E. \& Custódio, M. R. Aplysina Nardo (Porifera, Verongida, Aplysinidae) from the Brazilian coast with description of eight new species. Zootaxa 51, 1-51 (2007).

5. Alvarez, B., Crisp, M. D., Driver, F., Hooper, J. N. A. \& Van Soest, R. W. M. Phylogenetic relationships of the family axinellidae (Porifera: Demospongiae) using morphological and molecular data. Zool. Scr. 29, 169-198 (2000).

6. Alvarez, B. \& Hooper, J. N. A. Family Axinellidae Carter, 1875. Syst. Porifera 724-747 (2002) https://doi.org/10.1007/978-14615-0747-5_80.

7. Wörheide, G. \& Erpenbeck, D. DNA taxonomy of sponges - Progress and perspectives. J. Mar. Biol. Assoc. United Kingdom 87, 1629-1633 (2007).

8. Bell, J. J. The functional roles of marine sponges. Estuar. Coast. Shelf Sci. 79, 341-353 (2008).

9. Taylor, M. W., Radax, R., Steger, D. \& Wagner, M. Sponge-associated microorganisms: evolution, ecology, and biotechnological potential. Microbiol. Mol. Biol. Rev. 71, 295-347 (2007).

10. Thomas, T. et al. Diversity, structure and convergent evolution of the global sponge microbiome. Nat. Commun. 7, (2016).

11. Vacelet, J. \& Donadey, C. Electron microscope study of the association between some sponges and bacteria. J. Exp. Mar. Bio. Ecol. https://doi.org/10.1016/0022-0981(77)90038-7 (1977).

12. Moitinho-Silva, L. et al. The sponge microbiome project. Gigascience 6, (2017).

13. Muricy, G. Porifera in Catálogo Taxonômico da Fauna do Brasil. PNUD. Assessed at http://fauna.jbrj.gov.br/fauna/faunadobra sil/6in2020-13-10 (2020).

14. Muricy, G. et al. Integrative taxonomy widens our knowledge of the diversity, distribution and biology of the genus Plakina (Homosclerophorida: Plakinidae). Invertebr. Syst. 33, (2019).

15. Fortunato, H. F. M., Pérez, T. \& Lôbo-Hajdu, G. Morphological description of six species of Suberitida (Porifera: Demospongiae) from the unexplored north-eastern coast of Brazil, with emphasis on two new species. J. Mar. Biol. Assoc. United Kingdom https:// doi.org/10.1017/S0025315420000296 (2020).

16. Annunziata, B. B., Cavalcanti, T., Santos, G. G. \& Pinheiro, U. Two new Clathria (Axosuberites) Topsent, 1893 (Demospongiae: Poecilosclerida) from northeastern Brazil. Zootaxa 4671, 500-510 (2019).

17. Hardoim, C. C. P. et al. Diversity of bacteria in the marine sponge Aplysina fulva in brazilian coastal waters. Appl. Environ. Microbiol. 75, (2009).

18. Hardoim, C. C. P. et al. Diversity of the candidate phylum Poribacteria in the marine sponge Aplysina fulva. Brazilian J. Microbiol. 44, (2013).

19. Turque, A. S. et al. Environmental shaping of sponge associated archaeal communities. PLoS One 5, (2010).

20. Turque, A. S. et al. Bacterial communities of the marine sponges Hymeniacidon heliophila and Polymastia janeirensis and their environment in Rio de Janeiro Brazil. Mar. Biol. 155, 135-146 (2008).

21. Spalding, M. D. et al. Marine ecoregions of the world: a bioregionalization of coastal and shelf areas. Bioscience https://doi.org/ 10.1641/B570707 (2007).

22. Soares, M. de O. et al. Brazilian marine animal forests: a new world to discover in the southwestern Atlantic. Marine Anim. For. Ecol. Benthic Biodiver. Hotspots (2017). https://doi.org/10.1007/978-3-319-21012-4_51.

23. Custódio, M. R. \& Hajdu, E. Checklist de Porifera do Estado de São Paulo Brasil. Biota Neotrop. 11, 427-444 (2011).

24. Van Soest, R. W. M. Sponges of the Guyana Shelf. Zootaxa 4217, (2017).

25. Lavrov, D. V. et al. Mitochondrial DNA of Clathrina clathrus (Calcarea, Calcinea): Six linear chromosomes, fragmented rRNAs, tRNA editing, and a novel genetic code. Mol. Biol. Evol. https://doi.org/10.1093/molbev/mss274 (2013).

26. Erpenbeck, D., Hooper, J. N. A. \& Wörheide, G. CO1 phylogenies in diploblasts and the 'Barcoding of Life' - are we sequencing a suboptimal partition?. Mol. Ecol. Notes https://doi.org/10.1111/j.1471-8286.2005.01259.x (2006).

27. Erpenbeck, D. et al. Minimalist barcodes for sponges: a case study classifying African freshwater Spongillida. Genome https:// doi.org/10.1139/gen-2018-0098 (2019).

28. Voigt, O. \& Wörheide, G. A short LSU rRNA fragment as a standard marker for integrative taxonomy in calcareous sponges (Porifera: Calcarea). Org. Divers. Evol. https://doi.org/10.1007/s13127-015-0247-1 (2016).

29. Lamarão, F. R. M., Reis, E. C., Simao, T. A., Albano, R. M. \& Lôbo-Hajdu, G. Aplysina (Porifera: Demospongiae) species identification through SSCP-ITS patterns. J. Mar. Biol. Assoc. United Kingdom https://doi.org/10.1017/S0025315409991202 (2010).

30. Sperling, E. A., Rosengarten, R. D., Moreno, M. A. \& Dellaporta, S. L. The complete mitochondrial genome of the verongid sponge Aplysina cauliformis: Implications for DNA barcoding in demosponges. Hydrobiologia 687, 61-69 (2012).

31. Cleary, D. F. R. et al. The sponge microbiome within the greater coral reef microbial metacommunity. Nat. Commun. 10, 1-12. https://doi.org/10.1038/s41467-019-09537-8 (2019).

32. de Voogd, N. J., Gauvin-Bialecki, A., Polónia, A. R. M. \& Cleary, D. F. R. Assessing the bacterial communities of sponges inhabiting the remote western Indian Ocean island of Mayotte. Mar. Ecol. https://doi.org/10.1111/maec.12517 (2019).

33. Hardoim, C. C. P. et al. Effects of sample handling and cultivation bias on the specificity of bacterial communities in keratose marine sponges. Front. Microbiol. 5, (2014).

34. Simister, R. L., Deines, P., Botté, E. S., Webster, N. S. \& Taylor, M. W. Sponge-specific clusters revisited: a comprehensive phylogeny of sponge-associated microorganisms. Environ. Microbiol. 14, 517-524 (2012).

35. De Roy, K. et al. Environmental conditions and community evenness determine the outcome of biological invasion. Nat. Commun. (2013) https://doi.org/10.1038/ncomms2392.

36. Wittebolle, L. et al. Initial community evenness favours functionality under selective stress. Nature 458, 623-626 (2009).

37. Webster, N. S. \& Taylor, M. W. Marine sponges and their microbial symbionts: Love and other relationships. Environ. Microbiol. 14, 335-346 (2012).

38. Passarini, M. R. Z., Miqueletto, P. B., de Oliveira, V. M. \& Sette, L. D. Molecular diversity of fungal and bacterial communities in the marine sponge Dragmacidon reticulatum. J. Basic Microbiol. 55, 207-220 (2015).

39. Steinert, G., Rohde, S., Janussen, D., Blaurock, C. \& Schupp, P. J. Host-specific assembly of sponge-associated prokaryotes at high taxonomic ranks. Sci. Rep. 7, 1-9 (2017).

40. Steinert, G. et al. Prokaryotic diversity and community patterns in Antarctic continental shelf sponges. Front. Mar. Sci. 6, 1-15 (2019).

41. Indraningrat, A. A. G., Smidt, H. \& Sipkema, D. Bioprospecting sponge-associated microbes for antimicrobial compounds. Mar. Drugs https://doi.org/10.3390/md14050087 (2016). 
42. Taylor, M. W. et al. Sponge-specific bacteria are widespread (but rare) in diverse marine environments. ISME J. 7, 438-443 (2013).

43. Storey, M. A. et al. Metagenomic exploration of the marine sponge Mycale hentscheli uncovers multiple polyketide-producing bacterial symbionts. MBio 11, 1-16. https://doi.org/10.1128/mBio (2020).

44. Rix, L. et al. Heterotrophy in the earliest gut: a single-cell view of heterotrophic carbon and nitrogen assimilation in spongemicrobe symbioses. ISME J. 14, 2554-2567 (2020).

45. Engelberts, J. P. et al. Characterization of a sponge microbiome using an integrative genome-centric approach. ISME J. 14, $1100-1110(2020)$.

46. Bayer, K., Jahn, M. T., Slaby, B. M., Moitinho-Silva, L. \& Hentschel, U. Marine sponges as chloroflexi hot spots: genomic insights and high-resolution visualization of an abundant and diverse symbiotic clade. mSystems 3, 1-19 (2018).

47. Bayer, K., Scheuermayer, M., Fieseler, L. \& Hentschel, U. Genomic mining for novel FADH2-dependent halogenases in marine sponge-associated microbial consortia. Mar. Biotechnol. 15, 63-72 (2013).

48. Siegl, A. \& Hentschel, U. PKS and NRPS gene clusters from microbial symbiont cells of marine sponges by whole genome amplification. Environ. Microbiol. Rep. 2, 507-513 (2010).

49. Graham, E. D. \& Tully, B. J. Marine Dadabacteria exhibit genome streamlining and phototrophy-driven niche partitioning. bioRxiv 2020.06.22.165886 (2020).

50. Lin, W. et al. Expanding magnetic organelle biogenesis in the domain Bacteria. bioRxiv 2020.04.27.061960 (2020) https://doi. org/10.1101/2020.04.27.061960.

51. Ivanova, N. et al. Complete genome sequence of Truepera radiovictrix type strain (RQ-24 T). Stand. Genomic Sci. 4, 91-96 (2011).

52. Jin, M. et al. The diversity and commonalities of the radiation-resistance mechanisms of Deinococcus and its up-to-date applications. AMB Exp. 9, 1-12 (2019).

53. Hassan, F. M. N. \& Gupta, R. S. Novel sequence features of DNA repair genes/proteins from deinococcus species implicated in protection from oxidatively generated damage. Genes (Basel). 9, (2018).

54. Mueller, A. J. et al. Genomic and kinetic analysis of novel Nitrospinae enriched by cell sorting. bioRxiv (2020).

55. Astudillo-García, C. et al. Phylogeny and genomics of SAUL, an enigmatic bacterial lineage frequently associated with marine sponges. Environ. Microbiol. 20, 561-576 (2018).

56. Turon, M., Uriz, M. J., Martin, D. Multipartner symbiosis across biological domains: looking at the eukaryotic associations from a microbial perspective. $m$ Systems 4, 1-14 (2019). https://doi.org/10.1128/mSystems.

57. Yang, Y. et al. Roles of organohalide-respiring dehalococcoidia in carbon cycling. mSystems 5 (2020) https://doi.org/10.1128/ msystems.00757-19.

58. Gutleben, J. et al. Diversity of tryptophan halogenases in sponges of the genus Aplysina. FEMS Microbiol. Ecol. 95 (2019) https:// doi.org/10.1093/femsec/fiz108/5528619.

59. Lira, N. S. et al. Brominated compounds from marine sponges of the genus Aplysina and a compilation of their ${ }^{13} \mathrm{C}$ NMR spectral data. Mar. Drugs 9, 2316-2368 (2011).

60. Matturro, B., Frascadore, E. \& Rossetti, S. High-throughput sequencing revealed novel Dehalococcoidia in dechlorinating microbial enrichments from PCB-contaminated marine sediments. FEMS Microbiol. Ecol. 93, 1-10 (2017).

61. Wasmund, K. et al. Genome sequencing of a single cell of the widely distributed marine subsurface Dehalococcoidia, phylum Chloroflexi. ISME J. 8, 383-397 (2014).

62. Kohmoto, S. et al. Dragmacidin, a new cytotoxic bis(indole) alkaloid from a deep water marine sponge Dragmacidon sp. J. Org. Chem. https://doi.org/10.1021/jo00248a040 (1988).

63. Cruz, P. G., Martínez Leal, J. F., Daranas, A. H., Pérez, M., Cuevas, C. On the mechanism of action of dragmacidins I and J, two new representatives of a new class of protein phosphatase 1 and 2A inhibitors. ACS Omega 3, 3760-3767 (2018).

64. Whitman, W. B. et al. Proposal of the suffix -ota to denote phyla. Addendum to 'proposal to include the rank of phylum in the international code of nomenclature of prokaryotes'. Int. J. Syst. Evol. Microbiol. 68, 967-969 (2018).

65. Calisto, R. et al. Anticancer activity in Planctomycetes. Front. Mar. Sci. 5, 1-10 (2019).

66. Wiegand, S., Jogler, M. \& Jogler, C. On the maverick Planctomycetes. FEMS Microbiol. Rev. 42, 739-760 (2018).

67. Castelle, C. J. et al. Biosynthetic capacity, metabolic variety and unusual biology in the CPR and DPANN radiations. Nat. Rev. Microbiol. 16, 629-645 (2018).

68. León-Zayas, R. et al. The metabolic potential of the single cell genomes obtained from the Challenger Deep, Mariana Trench within the candidate superphylum Parcubacteria (OD1). Environ. Microbiol. 19, 2769-2784 (2017).

69. Nelson, W. C. \& Stegen, J. C. The reduced genomes of Parcubacteria (OD1) contain signatures of a symbiotic lifestyle. Front. Microbiol. 6, 1-14 (2015).

70. Allioux, M. et al. Genomic characterization and environmental distribution of a thermophilic anaerobe Dissulfurirhabdus thermomarina SH388T involved in disproportionation of sulfur compounds in shallow sea hydrothermal vents. Microorganisms 8 , $1-14(2020)$

71. Royalty, T. M. \& Steen, A. D. Quantitatively Partitioning Microbial Genomic Traits among Taxonomic Ranks across the Microbial Tree of Life. $m$ Sphere 4, (2019),

72. Huber, H., Hohn, M. J., Stetter, K. O. \& Rachel, R. The phylum Nanoarchaeota: Present knowledge and future perspectives of a unique form of life. Res. Microbiol. 154, 165-171 (2003).

73. Jarett, J. K. et al. Single-cell genomics of co-sorted Nanoarchaeota suggests novel putative host associations and diversification of proteins involved in symbiosis. Microbiome 6, 1-14 (2018).

74. Ghuneim, L. A. J., Jones, D. L., Golyshin, P. N. \& Golyshina, O. V. Nano-sized and filterable bacteria and archaea: Biodiversity and function. Front. Microbiol. 9, (2018).

75. St. John, E., Flores, G. E., Meneghin, J. \& Reysenbach, A. L. Deep-sea hydrothermal vent metagenome-assembled genomes provide insight into the phylum Nanoarchaeota. Environ. Microbiol. Rep. 11, 262-270 (2019).

76. McLachlan, J. Some considerations of growth of marine algae in artificial media. Can. J. Microbiol. 10, 769-772 (1964).

77. Hajdu, E., Peixinho, S. \& Fernandez, J. C. C. Esponjas marinhas da Bahia: Guia de campo e laboratório. Rio de Janeiro: Museu Nacional, Série Livros 45, 276 pp. (2011).

78. Lavrov, D. V., Wang, X. \& Kelly, M. Reconstructing ordinal relationships in the Demospongiae using mitochondrial genomic data. Mol. Phylogenet. Evol. 49, 111-124 (2008).

79. Altschul, S. F. et al. Gapped BLAST and PSI-BLAST: a new generation of protein database search programs. Nucleic Acids Res. 25, 3389-3402. https://doi.org/10.1093/nar/25.17.3389 (1997).

80. Altschul, S. F., Gish, W., Miller, W., Myers, E. W. \& Lipman, D. J. Basic local alignment search tool 2department of computer science. J. Mol. Biol. 215, 403-410 (1990).

81. Agarwala, R. et al. Database resources of the national center for biotechnology information. Nucleic Acids Res. 44, D7-D19 (2016).

82. Stecher G, Tamura K. Molecular evolutionary genetics analysis (MEGA) for macOS. Mol. Biol. Evol. 34, 1237-1239 (2020).

83. Kumar, S., Stecher, G., Li, M., Knyaz, C. \& Tamura, K. MEGA X: Molecular evolutionary genetics analysis across computing platforms. Mol. Biol. Evol. 35, 1547-1549 (2018).

84. Gouy, M., Guindon, S. \& Gascuel, O. Sea view version 4: a multiplatform graphical user interface for sequence alignment and phylogenetic tree building. Mol. Biol. Evol. 27, 221-224 (2010). 
85. Guindon, S. \& Gascuel, O. A simple, fast, and accurate algorithm to estimate large phylogenies by maximum likelihood. Syst. Biol. 52, 696-704 (2003).

86. Darriba, D., Taboada, G. L., Doallo, R. \& Posada, D. JModelTest 2: More models, new heuristics and parallel computing. Nat. Methods 9, 772 (2012).

87. Miller, M. A., Pfeiffer, W. \& Schwartz, T. Creating the CIPRES science gateway for inference of large phylogenetic trees. in 2010 Gateway Computing Environments Workshop, GCE 2010 (2010). https://doi.org/10.1109/GCE.2010.5676129.

88. Rodríguez, F., Oliver, J. L., Marín, A. \& Medina, J. R. The general stochastic model of nucleotide substitution. J. Theor. Biol. 142, 485-501 (1990).

89. Stamatakis, A. RAxML version 8: a tool for phylogenetic analysis and post-analysis of large phylogenies. Bioinformatics 30, 1312-1313 (2014).

90. Ronquist, F. \& Huelsenbeck, J. P. MrBayes 3: Bayesian phylogenetic inference under mixed models. Bioinformatics 19, 1572-1574 (2003).

91. Ronquist, F. et al. Mrbayes 3.2: efficient bayesian phylogenetic inference and model choice across a large model space. Syst. Biol. 61, 539-542 (2012).

92. Parada, A. E., Needham, D. M. \& Fuhrman, J. A. Every base matters: Assessing small subunit rRNA primers for marine microbiomes with mock communities, time series and global field samples. Environ. Microbiol. 18, 1403-1414 (2016).

93. Apprill, A., Mcnally, S., Parsons, R. \& Weber, L. Minor revision to V4 region SSU rRNA 806R gene primer greatly increases detection of SAR11 bacterioplankton. Aquat. Microb. Ecol. 75, 129-137 (2015).

94. Schloss, P. D. et al. Introducing mothur: Open-source, platform-independent, community-supported software for describing and comparing microbial communities. Appl. Environ. Microbiol. 75, 7537-7541 (2009).

95. Quast, C. et al. The SILVA ribosomal RNA gene database project: Improved data processing and web-based tools. Nucleic Acids Res. 41, D590-D596 (2013).

96. Yilmaz, P. et al. The SILVA and 'all-species Living Tree Project (LTP)' taxonomic frameworks. Nucleic Acids Res. 42, 643-648 (2014).

97. Edgar, R. C., Haas, B. J., Clemente, J. C., Quince, C. \& Knight, R. UCHIME improves sensitivity and speed of chimera detection. Bioinformatics 27, 2194-2200 (2011).

98. Oksanen, A. J. et al vegan: Community Ecology Package. R package version 2.5-6. https://CRAN.R-project.org/package=vegan (2019).

99. Hothorn, T., Bretz, F., Westfall, P. \& Heiberger, R. M. Package 'multcomp' title simultaneous inference in general parametric models. Biometrical J. (2016).

100. R Core Team. R: A language and environment for statistical computing. R Foundation for Statistical Computing, Vienna, Austria. https://www.R-project.org/. (2020).

101. Chen, H. \& Boutros, P. C. VennDiagram: a package for the generation of highly-customizable Venn and Euler diagrams in R. BMC Bioinformatics 15, 35. https://doi.org/10.1186/1471-2105-12-35 (2011).

102. Segata, N. et al. Metagenomic biomarker discovery and explanation. Genome Biol. 12, (2011).

103. Afgan, E. et al. The galaxy platform for accessible, reproducible and collaborative biomedical analyses: 2018 update. Nucleic Acids Res. 46, W537-W544 (2018).

104. Wemheuer, F. et al. Tax4Fun2: prediction of habitat-specific functional profiles and functional redundancy based on $16 \mathrm{~S}$ rRNA gene sequences. Environ. Microbiome 15, 11 (2020).

\section{Acknowledgements}

This research was financed by the Fundação de Amparo à Pesquisa do Estado de São Paulo (FAPESP) through the Young Investigator Project Number 2016/17189-7 granted to Cristiane C.P. Hardoim. Cristiane C. P. Hardoim was also the recipient of a Young Investigator fellowship granted by FAPESP (No. 2017/10157-5). Andressa C.M. Ramaglia was the recipient of a Technical Training fellowship (TT-3) granted by FAPESP (No. 2018/12937-0). We would like to thank the CEBIMar-USP for allowing the use of infrastructure, and also the staff, especially Eduardo Honuma and Joseilto Medeiros de Oliveira for their assistance with sampling and Emerson de Paula Candido for the bureaucratic organization. We also would like to thank Marcelo U. Ferreira for the acquisition of the Boto Server (FAPESP 2017/11380-0) and João Marcelo Pereira Alves for allowing its use and assistance to run metagenomics analyses from Institute of Biomedical Science - USP. We are also very thankful to Ulisses Pinheiro from UFPE for providing Aplysina spp. samples, and Bernd Wemheuer from the Institute of Microbiology and Genetics (Georg-August University of Göttingen, Germany) for his aid with Tax4fun2. We would like to thank Thiago S. de Paula from Universidade do Estado do Rio de Janeiro for the assistance with submission of cytochrome b sequences.

\section{Author contributions}

C.C.P.H. designed the experiment. C.C.P.H. and A.C.M.R. collected and pre-processed the samples. C.C.P.H. prepared samples for metagenomic sequencing and performed all data analysis. M.R.C. and G.L-H. identified the sponge species. C.C.P.H. wrote the manuscript with feedbacks from M.R.C. and G.L-H. All authors approved the content of the manuscript.

\section{Competing interests}

The authors declare no competing interests.

\section{Additional information}

Supplementary Information The online version contains supplementary material available at https://doi.org/ 10.1038/s41598-021-88288-3.

Correspondence and requests for materials should be addressed to C.C.P.H.

Reprints and permissions information is available at www.nature.com/reprints.

Publisher's note Springer Nature remains neutral with regard to jurisdictional claims in published maps and institutional affiliations. 
(c) (i) Open Access This article is licensed under a Creative Commons Attribution 4.0 International cc) License, which permits use, sharing, adaptation, distribution and reproduction in any medium or format, as long as you give appropriate credit to the original author(s) and the source, provide a link to the Creative Commons licence, and indicate if changes were made. The images or other third party material in this article are included in the article's Creative Commons licence, unless indicated otherwise in a credit line to the material. If material is not included in the article's Creative Commons licence and your intended use is not permitted by statutory regulation or exceeds the permitted use, you will need to obtain permission directly from the copyright holder. To view a copy of this licence, visit http://creativecommons.org/licenses/by/4.0/.

(C) The Author(s) 2021 